\title{
Spectral analysis of WC stars in M 33 using CFHT-MOS
}

\author{
Jay B. Abbott ${ }^{1}$, Paul A. Crowther ${ }^{1}$, Laurent Drissen ${ }^{2}$, Luc Dessart ${ }^{3}$, \\ and Pierre Martin ${ }^{4}$ \\ ${ }^{1}$ Department of Physics and Astronomy, University College London, \\ Gower Street, London WC1E 6BT, UK \\ ${ }^{2}$ Département de Physique, Université Laval, Québec, G1K 7P4, Canada \\ ${ }^{3}$ Sterrekundig Instituut, Universiteit Utrecht, \\ Princetonplein 5, NL-3584 CC Utrecht, Nederland \\ ${ }^{4}$ Canada-France-Hawai' $i$ Telescope Corporation, \\ Waimea Headquarters, PO Box 1597, Kamuela, HI 96743, USA
}

\begin{abstract}
We have analyzed eight single WC4-7 stars in M 33, spanning a variety of galactocentric distances, using optical CFHT-MOs spectroscopy. Pro visional stellar parameters are determined using the code of Hillier \& Miller (1998), which are compared here to Galactic and LMC counterparts.
\end{abstract}

\section{Introduction and observations}

Studies of young, massive stars in M33 provide a useful test of evolutionary models. This is because its metallicity spans $1.0 \mathrm{Z}_{\odot}$ (inner regions) to $0.2 \mathrm{Z}_{\odot}$ (outer spiral arms), it is nearby, with a well determined distance, a low extinction, and is viewed with a low inclination. Observations used in this present study were obtained using the Multi-object spectrograph (MOS) on the $3.6 \mathrm{~m}$ Canada France Hawaii Telescope (CFHT) between October 2000 and October 2001. 50 Wolf-Rayet stars were observed, representing approximately one third of the known WR content of M 33. From this sample, eight apparently single WC stars were selected and the photometry for each star was calculated from a $V$-band CFHT $12 \mathrm{~K}$ image of M 33, using nearby secondary standards taken from Macri et al. (2001), whilst archival HST-wFPC2 images were used for two stars.

\section{Results}

There have already been a number of detailed studies analyzing Galactic and LMC WC-type stars (e.g., Crowther et al. 2002) using CMFGEN (Hillier \& Miller $1998)$ to calculate stellar properties $\left(v_{\infty}, \dot{M}, T, \log L\right.$, abundances). For our sample we used identical analysis techniques. $\mathrm{C} / \mathrm{He}$ ratios were determined from the diagnostic lines of He II 5412 and CIV 5471. For the oxygen abundance the main optical diagnostic line of $\mathrm{O}$ III/V 5592 was used. The range of $(\mathrm{C}+\mathrm{O}) / \mathrm{He}$ ratios derived for this study are comparable to those previously found for Galactic and LMC WC stars, as illustrated in Figure 1 (right panel). AM 16 (WC5) and AM 17 (WC5) appear to be rich in oxygen, having $\mathrm{C} / \mathrm{O} \simeq 1$ (by number). 

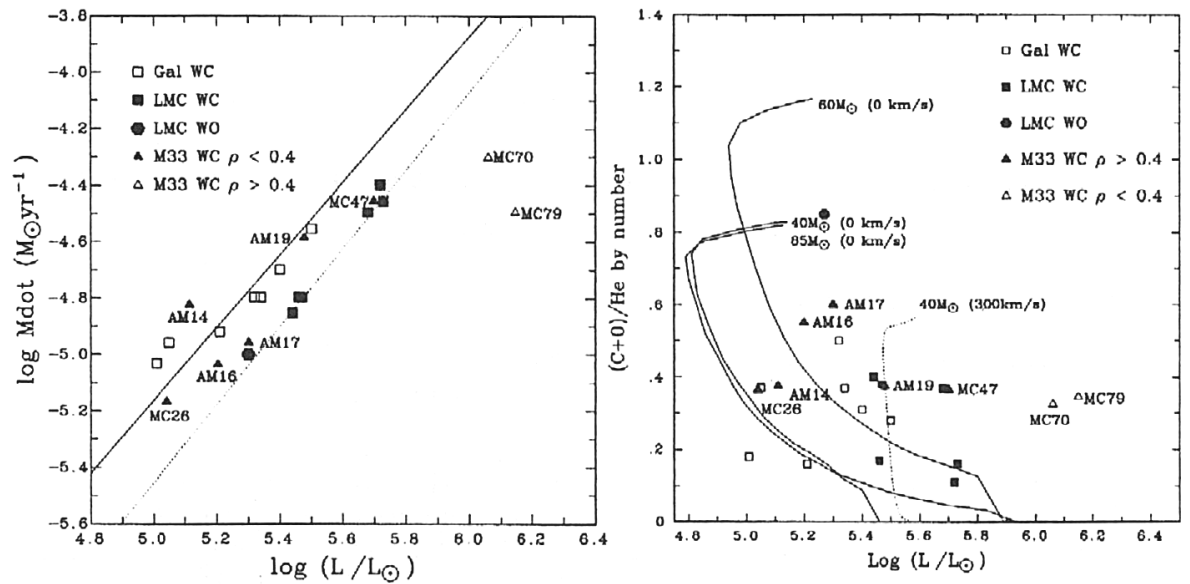

Figure 1. Left: Comparison between mass-loss rates and luminosities of Galactic, LMC and M 33 WC stars. The lines correspond to the calibrations of Nugis \& Lamers (2000) for Galactic WR stars (solid), plus a linear fit to the LMC data (dotted, Crowther et al. 2002). Right: Comparison between $(\mathrm{C}+\mathrm{O}) / \mathrm{He}$ values for WC stars in the Galaxy, LMC and M 33 vs. luminosities. The evolutionary predictions shown are for non-rotating stars (solid, Meynet et al. 1994), and for an initially rapidly rotating $40 \mathrm{M}_{\odot}$ model (dotted, Meynet \& Maeder 2000), all at solar metallicity.

Luminosities of M33 WC stars span a much greater range than LMC or Galactic counterparts, i.e., $5.0 \leq \log L / \mathrm{L}_{\odot} \leq 6.2$. Figure 1 (left panel) compares mass-loss rates of M33 WC stars with previously studied LMC and Galactic WC stars. We find that M 33 stars lying closer than $40 \%$ of the Holmberg radius, with metallicities $\geq 0.6 \mathrm{Z}_{\odot}$, possess stellar winds which are comparable in strength to LMC and Galactic stars. Much weaker winds are found for MC70 (WC5) and particularly MC79 (WC4), with SMC-like metallicities. This fits in with the recent suggestion by Crowther et al. (2002) of a metallicity dependence for WC winds, with $\dot{M} \propto Z^{0.5}$.

This work is part of an on-going project to study WR stars at a number of different metallicities, ranging from metal-poor galaxies, such as IC 10, to metal-rich galaxies like, M 83 (see Crowther et al. , these Proceedings).

Acknowledgments. We wish to thank John Hillier for the use of his code.

\section{References}

Crowther, P.A., Dessart, L., Hillier, D.J., et al. 2002, A\&A 392, 653

Hillier, D.J., Miller, D.L., 1998, ApJ 496, 407

Macri, L.M., Stanek, K.Z., Sasselov, D.D., et al. 2001, AJ 121, 861

Meynet, G., Maeder, A., Schaller, G., et al. 1994, A\&AS 103, 97

Meynet, G., Maeder, A., 2000, A\&A 361, 101

Nugis, T., Lamers, H., 2000, A\&A 360, 227 